\title{
Author Correction: Cytochrome P450 oxidoreductase contributes to phospholipid peroxidation in ferroptosis
}

Yilong Zou, Haoxin Li, Emily T. Graham (D, Amy A. Deik, John K. Eaton D, Wenyu Wang, Gerardo Sandoval-Gomez, Clary B. Clish D, John G. Doench (D) and Stuart L. Schreiber (D)

Correction to: Nature Chemical Biology https://doi.org/10.1038/s41589-020-0472-6, published online 17 February 2020

In the version of this Article originally published, there was an error in the catalog information for one antibody in the "immunoblot analysis" section of the Methods. In the sentence "Membranes were blocked with $50 \%$ Odyssey blocking buffer (LiCor) diluted with $0.1 \%$ Tween-20-containing Tris buffered saline (TBST) and immunoblotted with antibodies against POR (Abcam, ab133303, rabbit monoclonal antibody, clone no. UOTR1B493, lot no. GR290350- 7, used at 1:1,000 dilution), GPX4 (Abcam, ab41787, rabbit polyclonal antibody, lot no. GR56784-1, used at 1:1,000 dilution) and $\beta$-actin (8H10D10, no. 3700 and 13E5, no. 4970, Cell Signaling Technologies, used at 1:5,000 dilution)," the information for the POR antibodies should be "Abcam, ab180597, $0.076 \mathrm{mg} / \mathrm{mL}$, Rabbit mAb to Cytochrome P450 reductase, clone number EPR 14479(B), Lot number GR148691-9, used at 1:1,000 dilution”. The errors have been corrected.

Published online: 2 March 2021

https://doi.org/10.1038/s41589-021-00767-w

(c) The Author(s), under exclusive licence to Springer Nature America, Inc. 2021

\section{Retraction Note: Orphan receptor ligand discovery by pickpocketing pharmacological neighbors}

Tony Ngo, Andrey V. Ilatovskiy, Alastair G. Stewart, James L. J. Coleman, Fiona M. McRobb, R. Peter Riek, Robert M. Graham, Ruben Abagyan, Irina Kufareva and Nicola J. Smith

Retraction of: Nature Chemical Biology https://doi.org/10.1038/nchembio.2266, published online 19 December 2016

In this paper, we developed GPCR-CoINPocket, a computational method for identifying pharmacological similarities of GPCRs, including orphan receptors, solely from their sequences. Guided by our GPCR-CoINPocket predictions for the orphan receptor GPR37L1, we reported several compounds as inverse agonists of GPR37L1 constitutive G $\alpha_{s}$-directed activity. Unfortunately, after the work was published, we discovered a cloning error in the GPR37L1 construct used for compound screening in Fig. 5 and Supplementary Figs. 5 and 6. The corrected GPR37L1 construct no longer displays constitutive activity, and the predicted compounds do not evoke a GPR37L1-mediated response in the pharmacological assays tested (refer to the accompanying Matters Arising). Therefore, we are retracting this study, and all authors agree with this decision. We sincerely apologize for any impact on the scientific community. The computational method for identifying pharmacological neighbors, GPCR-CoINPocket, is unaffected by this error.

Published online: 1 March 2021

https://doi.org/10.1038/s41589-021-00746-1

(c) The Author(s), under exclusive licence to Springer Nature America, Inc. 2021 